in this study, we conclude that PFO device closure should be offered cautiously and only after careful multi-disciplinary assessment.

Conflict of Interest None to declare

\section{UPDATE ON FAMILIAL THORACIC AORTIC ANEURYSM DISEASE IN THE 100,000 GENOMES PROJECT: SPACE FOR DISCOVERY}

${ }^{1}$ Catherine Francis*, ${ }^{2}$ Yalda Jamshidi, ${ }^{3}$ George Gkoutos, ${ }^{4}$ Bernard Keavney, ${ }^{5}$ Paul Clift, ${ }^{6}$ Tom Fowler. 'National Heart \& Lung Institute, Imperial College London; ${ }^{2}$ Genetics Research Centre, Molecular \& Clinical Sciences Institute, St George's University of London; ${ }^{3}$ Institute of Cancer and Genomic Sciences, University of Birmingham; ${ }^{4}$ Faculty of Biology, Medicine and Health, University of Manchester; ${ }^{5}$ Department of Cardiology, Queen Elizabeth Hospital Birmingham; ${ }^{6}$ Genomics England, London, UK

\subsection{6/heartjnl-2019-BCS.139}

Background The 100,000 Genomes Project offers an unprecedented opportunity to leverage whole genome sequencing data to characterise the hereditary basis of Familial Thoracic Aortic Aneurysm Disease (FTAAD). FTAAD is a heterogeneous group of syndromic and isolated aortopathies. The genetic basis of this presentation often remains undiscovered.

Purpose To define the FTAAD cohorts available in the 100,000 Genomes Project and assess the current diagnostic yield and space for future discovery.

Materials and methods As of February 2019, 536 probands with FTAAD and HPO terms for thoracic aortic disease have been recruited to the 100,000 Genomes Project. Inclusion criteria included:

- Thoracic aortopathy $<50$ years with no additional risk factors or

- Marfan syndrome with no identified FBN1 mutation or

- Loeys-Dietz syndrome or related condition, and

- Negative initial genetic screening to include FBN1, TGFBR1\&2, ACTA2 where appropriate.

Whole genome sequencing, quality control and filtering was carried out by Genomics England as previously
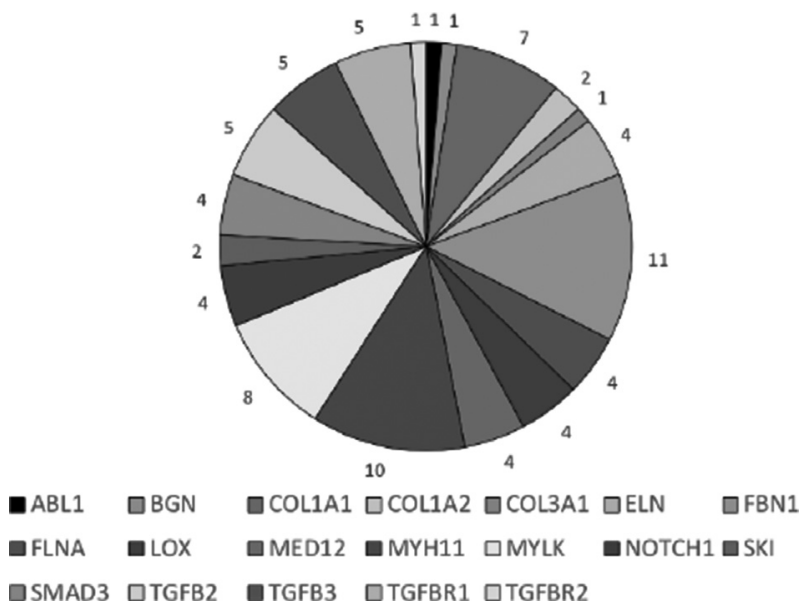

Abstract 142 Figure 1 Genetic heterogeneity of FTAAD: Pie chart showing validated aortopathy genes in which rare, protein-altering variants are found

described. Aortopathy genes are defined in PanelApp, an expert-curated gene list with validated associations with FTAAD. Genes curated for only skeletal phenotypes (eg FBN2, FLCN) with no evidence for aortic involvement were excluded. Tier 1 variants are rare and protein-truncating; tier 2 variants are rare and protein-altering. Tiered variants were excluded if Clinvar status was benign, or if they were not fully penetrant.

Results The cohort has a mean age of 44 years (range 0-79). 110 probands were $<30$ years old at disease onset. 113 probands had a history of aortic dissection. 96 had additional aortic valve abnormalities, with 36 reported as bicuspid. Only 22 were reported to have significant AR or AS. Skeletal abnormalities were prevalent, with 66 having sternal abnormalities.

Only 8 Tier 1 variants in validated aortopathy genes were reported in this cohort. These were in BGN, FBN1, LOX, MYH11, MYLK, TGFB2 and TGFBR1 (see table1). This

Abstract 142 Table 1 Variants in curated aortopathy gene panel identified in FTAAD probands

\begin{tabular}{|c|c|c|c|}
\hline \multicolumn{4}{|c|}{ TIER1 (protein-truncating rare variants) } \\
\hline Gene & Variant (GRCh38) & Consequence & $\begin{array}{c}\text { Pathogenicity (ClinVar or } \\
\text { clinical team) }\end{array}$ \\
\hline LOX & $\begin{array}{l}5: 122070160 T>T A \\
5: 122077495 T C>T\end{array}$ & $\begin{array}{l}\text { Frameshift } \\
\text { Frameshift }\end{array}$ & $\begin{array}{l}\text { Likely Pathogenic } \\
\text { Likely Pathogenic }\end{array}$ \\
\hline FBN1 & $15: 48645574 C>T$ & Splice donor (LoF) & Not reported \\
\hline BGN & $X: 153508247 G>A$ & $\begin{array}{c}\text { Splice acceptor } \\
\text { (LoF) }\end{array}$ & Likely pathogenic \\
\hline TGFBR1 & 9:99146492GC>G & Frameshift & Not reported \\
\hline TGFB2 & $1: 218441264 C>T$ & Stop gained & $\begin{array}{l}\text { Likely pathogenic (similar } \\
\text { variants same codon) }\end{array}$ \\
\hline MYH11 & $16: 15721419 \mathrm{~T}>\mathrm{TA}$ & Frameshift & $\begin{array}{l}\text { Likely pathogenic; } \\
\text { de novo }\end{array}$ \\
\hline MYLK & $3: 12368222 A C T>A$ & Frameshift & Likely pathogenic \\
\hline
\end{tabular}


defines a diagnostic yield of just $8 / 536(6 \%)$ in this patient group, using known aortopathy gene panels. Surprisingly, none of these variants were found in probands under 30 at disease onset.

There were 68 classic trios in this cohort (proband + mother and father). However, just one of these has a clearly pathogenic de novo variant identified (in MYH11). There were just 2 Tier 2 variants of uncertain significance (VUS) in aortopathy genes in other trios. 10 larger family structures have yielded no clear pathogenic candidates to date. The majority of the cohort are recruited as singletons.

75 Tier 2 variants have been identified to date in 19 aortopathy genes (see figure 1). Just 1 of these (in LOX) can be classified as likely pathogenic. The remainder are VUSs.

Conclusion In this genetically pre-screened cohort with FTAAD, genetic diagnosis remains elusive. The variety of genes in which protein-altering variants are found highlights the genetic heterogeneity of this condition. We plan further systematic studies of the family structures available in the 100,000 Genomes Project and case:control studies to further elucidate the genetic architecture of FTAAD. The negative findings to date, particularly in probands with young-onset aortopathy, make it likely that responsible genes are yet to be discovered in many cases.

This research was made possible through access to the data and findings generated by the 100,000 Genomes Project.

Conflict of Interest None

\section{Acute Coronary Syndromes}

\section{A PIVOTAL ROLE FOR NRF2 IN ENDOTHELIAL DETACHMENT- IMPLICATIONS FOR ENDOTHELIAL EROSION OF STENOTIC PLAQUES}

\begin{abstract}
'Sandro Satta*, ${ }^{2}$ Michael McElroy, ${ }^{1}$ Alex Langford-Smith, ' ${ }^{1}$ lenn Ferris, ${ }^{3}$ Jack Teasdale, ${ }^{4}$ Yongcheol Kim, ${ }^{5}$ Giampaolo Niccoli, ${ }^{6}$ Ajime Tanjeko, ${ }^{7}$ Jef Serré, ${ }^{3}$ Georgina Hazell, ${ }^{3}$ Graciela Sala-Newby, ${ }^{1}$ Ping Wang, ${ }^{3}$ Jason Johnson, ${ }^{1}$ Martin Humphries, ${ }^{7}$ Ghislaine GayanRamirez, ${ }^{8}$ Peter Libby, ${ }^{5}$ Filippo Crea, ${ }^{1}$ Hans Degens, ${ }^{9}$ Frank Gijsen, ${ }^{10}$ Tom Johnson, ${ }^{1}$ Amir Keshmiri, ${ }^{1} Y$ vonne Alexander, ${ }^{3}$ Andrew Newby, ${ }^{1}$ Stephen White. ${ }^{1}$ Manchester Metropolitan University; ${ }^{2}$ University of Manchester; ${ }^{3}$ University of Bristol; ${ }^{4}$ Chonnam National University Hospital; ${ }^{5}$ Catholic University of the Sacred Heart - Rome; ${ }^{6}$ KULeuven; ${ }^{7}$ KULeuven; ${ }^{8}$ Harvard Medical School; ${ }^{9}$ Erasmus MC; ${ }^{10}$ Bristol Heart Institute, Bristol Royal Infirmary
\end{abstract}

\subsection{6/heartjnl-2019-BCS.140}

Introduction Endothelial erosion of atherosclerotic plaques and resulting thrombosis causes approximately 30\% of acute coronary syndromes (ACS). Plaque erosion is most frequently observed in smokers, which induces endothelial dysfunction, partially through elevated circulating mediators of inflammation, such as tumour necrosis factor-alpha (TNF $\alpha$ ), as well as free radical, oxidative and chemically induced damage. We have previously demonstrated that fresh aqueous cigarette smoke extract (CSE) increases the expression of Nrf2-target genes in human coronary artery endothelial cells, which was further increased by TNF $\alpha$ in a shear stress-dependent manner.
Methods The haemodynamic environment significantly regulates both plaque development and endothelial function, therefore we determined the haemodynamic environment permissive for plaque erosion. We reconstructed the coronary artery geometries from 17 heart attack patients with thrombi overlying intact fibrous caps (OCT-defined erosion) and performed computational fluid dynamic analysis. We created an in vitro model of erosion by culturing human coronary artery endothelial cells under elevated flow and exposing them to CSE and TNF $\alpha$.

Results We identified that in 14 cases of OCT-defined erosion occurred in areas of stenosis, with the preeminent flow feature being elevated flow. Exposing human coronary artery endothelial cells to elevated flow, CSE and TNF $\alpha$ induced significant endothelial detachment, which was enhanced by pharmacological activation of the antioxidant system controlled by transcription factor Nrf2. The Oxidative Stress Growth INhibitor genes OSGIN1 and OSGIN2 were both maximally upregulated under these conditions and also in the aortas of mice exposed to cigarette smoke. Adenoviral overexpression of OSGIN1+2 in static culture resulted in cell cycle arrest in S-phase (5.5-fold increase, $\mathrm{p}=0.003)$, with a significant increase in the number of multinucleated cells (4.5-fold, $p=<0.001)$. Immunocytochemical analysis indicated loss of focal adhesions and stress fibres, dysregulation of autophagy and induction of senescence in HCAEC, with a significant increase in senescence-associated $\beta$-galactosidase staining (6.7-fold, $\mathrm{p}=<0.001)$ and P16 expression (3.2-fold, $\mathrm{p}=0.035)$. Importantly, overexpression of either $\mathrm{Nrf} 2$, or OSGIN1+2 induced cell detachment, which was independent of apoptosis, and could be rescued by inhibition of HSP70 nucleotide binding site using VER-155008, or AMPK activation using Metformin.

Conclusions In summary, we have defined the haemodynamic environment in which endothelial erosion occurs and identified that smoking-induced hyperactivation of Nrf2 may promote endothelial cell detachment, contributing to plaque erosion overlying stenotic plaques, through the combined upregulation of OSGIN1 and OSGIN2. This highlights a completely novel mechanism potentially contributing to $30 \%$ of ACS and suggests possible therapeutic avenues for further investigation.

Conflict of Interest none

\section{HIGH-SENSITIVITY CARDIAC TROPONIN AND THE FOURTH UNIVERSAL DEFINITION OF MYOCARDIAL INFARCTION}

${ }^{1}$ Andrew Chapman*, ${ }^{2}$ Philip Adamson, ${ }^{1}$ Anoop Shah, ${ }^{1}$ Atul Anand, ${ }^{1}$ Fiona Strachan, ${ }^{1}$ Kuan Ken Lee, ${ }^{1}$ Amy Ferry, ${ }^{3}$ Dennis Sandeman, ${ }^{1}$ Catherine Stables, ${ }^{4}$ David Newby, ${ }^{1}$ Nicholas Mills. ${ }^{1}$ University of Edinburgh; ${ }^{2}$ University of Otago, Christchurch; ${ }^{3}$ NHS Fife; ${ }^{4}$ British Heart Foundation Centre for Cardiovascular Science, University of Edinburgh

\subsection{6/heartjnl-2019-BCS.141}

Background The Universal Definition of Myocardial Infarction recommends the 99th centile diagnostic threshold using a high-sensitivity cardiac troponin (hs-cTn) assay and 